\title{
Supporting Information for Computational Screening of MOFs and Zeolites for Direct Air Capture of Carbon Dioxide under Humid Conditions
}

\author{
John M. Findley ${ }^{1}$ and David S. Sholl ${ }^{1 \ddagger}$ \\ ${ }^{1}$ School of Chemical and Biomolecular Engineering, Georgia Institute of Technology, \\ Atlanta, Georgia 30332-0100, United States \\ $\$+1-404-894-2822$, david.sholl@chbe.gatech.edu
}




\section{Attachments:}

RASPA force field definition files for D2FF for A1PO/GaPO zeolites are included in folders named D2FF_AlPO and D2FF_GaPO. The parameters are also shown in Tables S.1 - S.4.

$\mathrm{CO}_{2}$ and $\mathrm{H}_{2} \mathrm{O}$ heats of adsorption, and Pore-Limiting Diameters (PLDs) for materials screened are located in Zeolite_Screening.csv, CoREMOF_Step1.csv ( $\mathrm{CO}_{2}$ heats of adsorption), and CoREMOF_Step2.csv ( $\mathrm{H}_{2} \mathrm{O}$ heats of adsorption). This data was presented in Figure 1.

A spreadsheet containing tabulated adsorption isotherm data from Figures 3 and 5 is located in Isotherms.xlsx.

\section{D2FF Fitting}

\begin{tabular}{c|c|c}
\hline Cross-Species & $\boldsymbol{\varepsilon}(\mathbf{K})$ & $\boldsymbol{\sigma}(\AA)$ \\
\hline Oz-C_co2 & 30.07 & 3.200 \\
Oz-O_co2 & 24.20 & 3.080 \\
P - C_co2 & 57.22 & 3.508 \\
P - O_co2 & 45.77 & 3.386 \\
Al - C_co2 & 77.20 & 3.435 \\
Al - O_co2 & 59.90 & 3.313 \\
Oz-O_SPCE & 41.72 & 3.385 \\
P - O_SPCE & 83.24 & 3.374 \\
Al-O_SPCE & 111.4 & 3.301
\end{tabular}

Table S1 - The D2FF Lennard-Jones parameters for $\mathrm{CO}_{2}$ and $\mathrm{H}_{2} \mathrm{O}$ in AlPO zeolites.

\begin{tabular}{c|c|c}
\hline Cross-Species & $\boldsymbol{\varepsilon}(\mathbf{K})$ & $\boldsymbol{\sigma}(\boldsymbol{\AA})$ \\
\hline Oz - C_co2 & 30.07 & 3.200 \\
Oz-O_co2 & 24.20 & 3.080 \\
P - C_co2 & 58.02 & 3.612 \\
P - O_co2 & 45.40 & 3.486 \\
Ga - C_co2 & 94.91 & 3.549 \\
Ga-O_co2 & 74.55 & 3.423 \\
Oz-O_SPCE & 41.72 & 3.385 \\
P - O_SPCE & 149.1 & 3.116 \\
Ga-O_SPCE & 244.8 & 3.060
\end{tabular}

Table S2 - The D2FF Lennard-Jones parameters for $\mathrm{CO}_{2}$ and $\mathrm{H}_{2} \mathrm{O}$ in $\mathrm{GaPO}$ zeolites. 


\begin{tabular}{c|c|}
\hline Species & Charge $(|\mathbf{e}|)$ \\
\hline O (Ga-O-Ga) & -0.8715 \\
O (Ga-O-P) & -0.8965 \\
P & 1.843 \\
Ga & 1.743 \\
O_co2 & -0.3256 \\
C_co2 & 0.6512 \\
O_SPCE & -0.8476 \\
H_SPCE & 0.4238
\end{tabular}

Table S3 - The DDEC3 point charges in GaPO zeolites.

\begin{tabular}{c|c|}
\hline Species & Charge $(|\mathbf{e}|)$ \\
\hline O (Al-O-Al) & -1.079 \\
O (Al-O-P) & -1.0655 \\
P & 2.104 \\
Al & 2.158 \\
O_co2 & -0.3256 \\
C_co2 & 0.6512 \\
O_SPCE & -0.8476 \\
H_SPCE & 0.4238
\end{tabular}

Table S4 - The DDEC3 point charges in AlPO zeolites. 


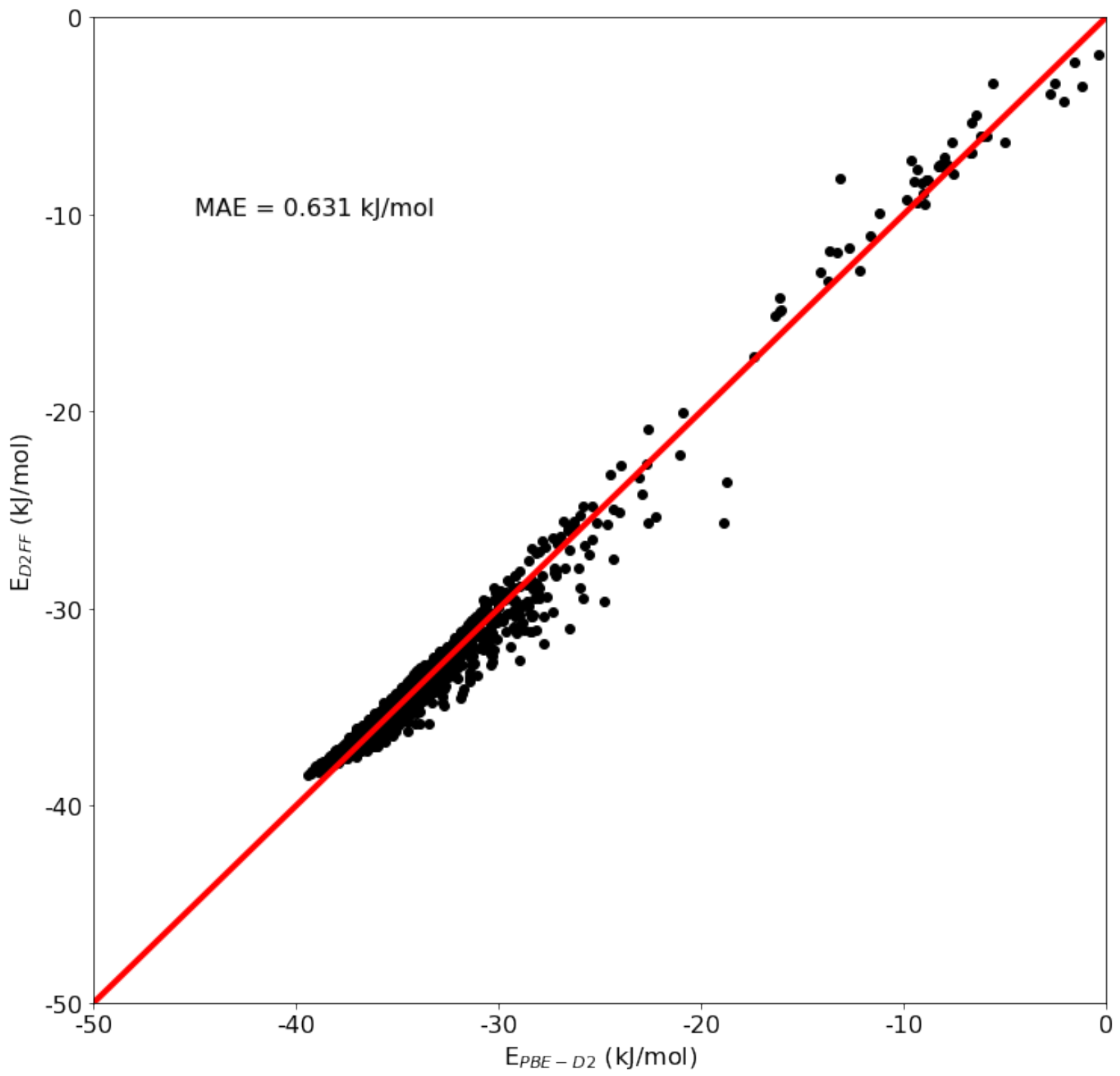

Figure S1 - A parity plot showing comparing interaction energies for D2FF and PBE-D2 for $\mathrm{CO}_{2}$ in an AlPO zeolite with the DFT topology. 


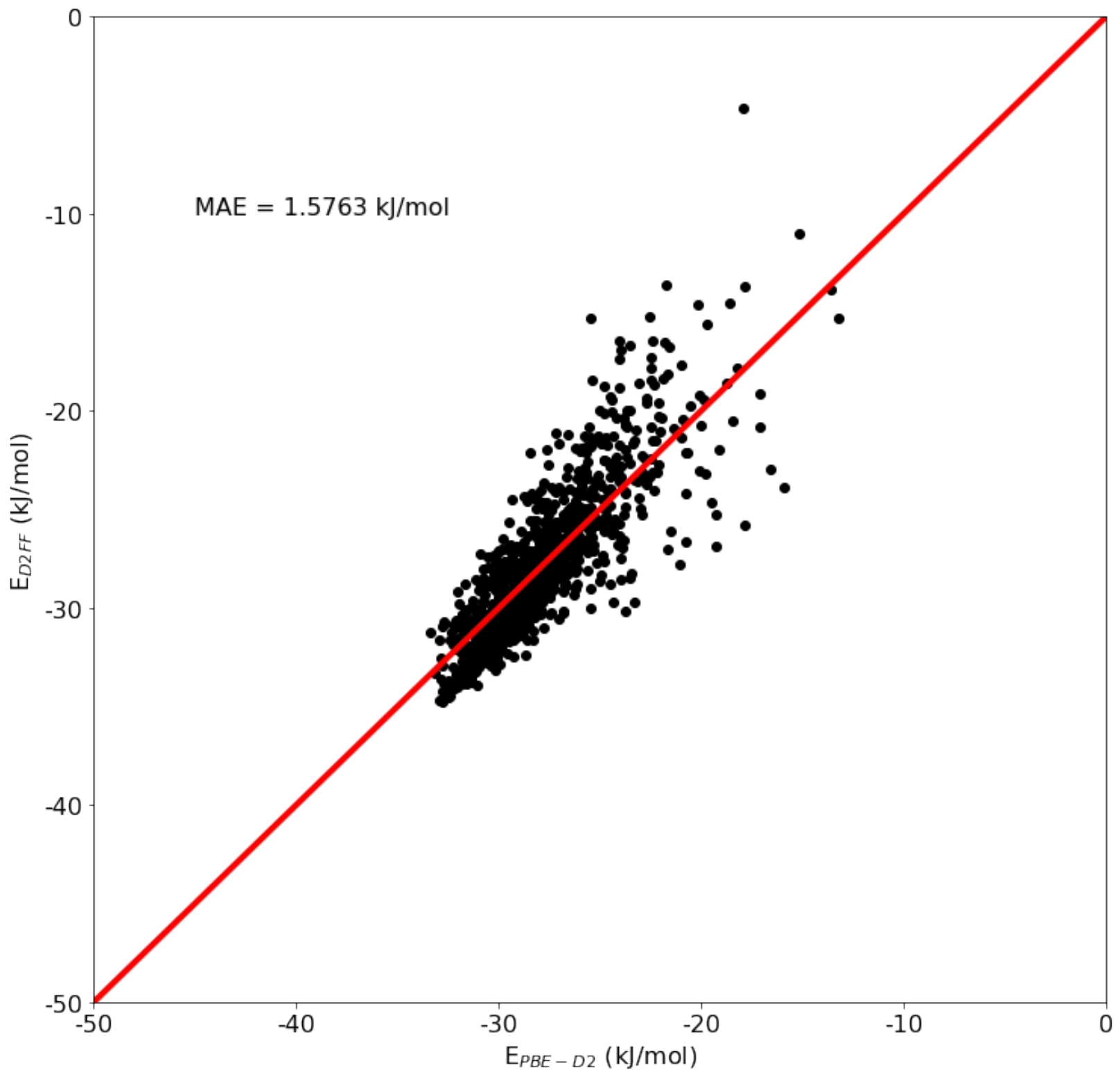

Figure S2 - A parity plot showing comparing interaction energies for D2FF and PBE-D2 for $\mathrm{H}_{2} \mathrm{O}$ in an AlPO zeolite with the DFT topology. 


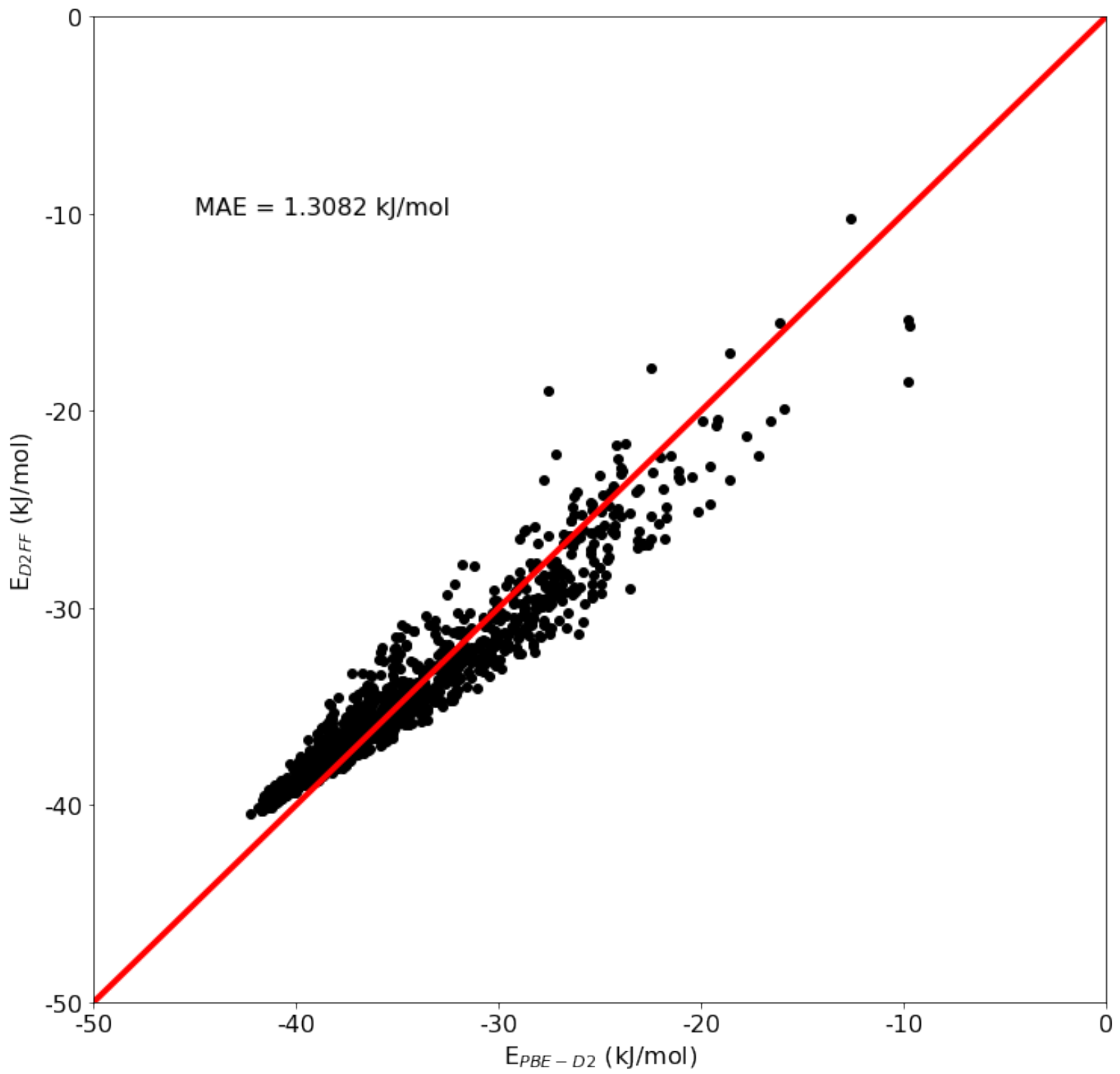

Figure S3 - A parity plot showing comparing interaction energies for D2FF and PBE-D2 for $\mathrm{CO}_{2}$ in an GaPO zeolite with the DFT topology. 


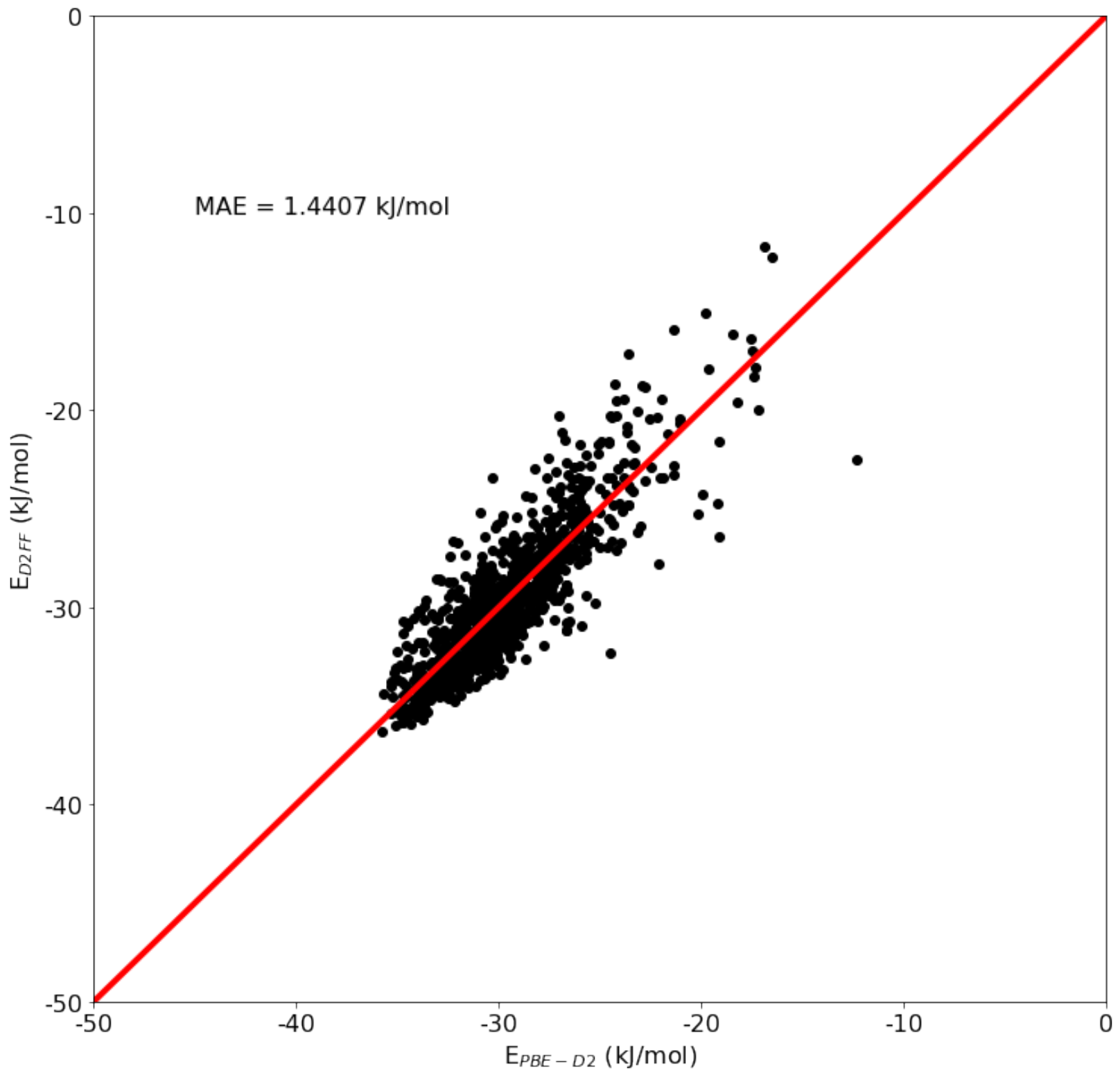

Figure S4 - A parity plot showing comparing interaction energies for D2FF and PBE-D2 for $\mathrm{H}_{2} \mathrm{O}$ in an GaPO zeolite with the DFT topology. 


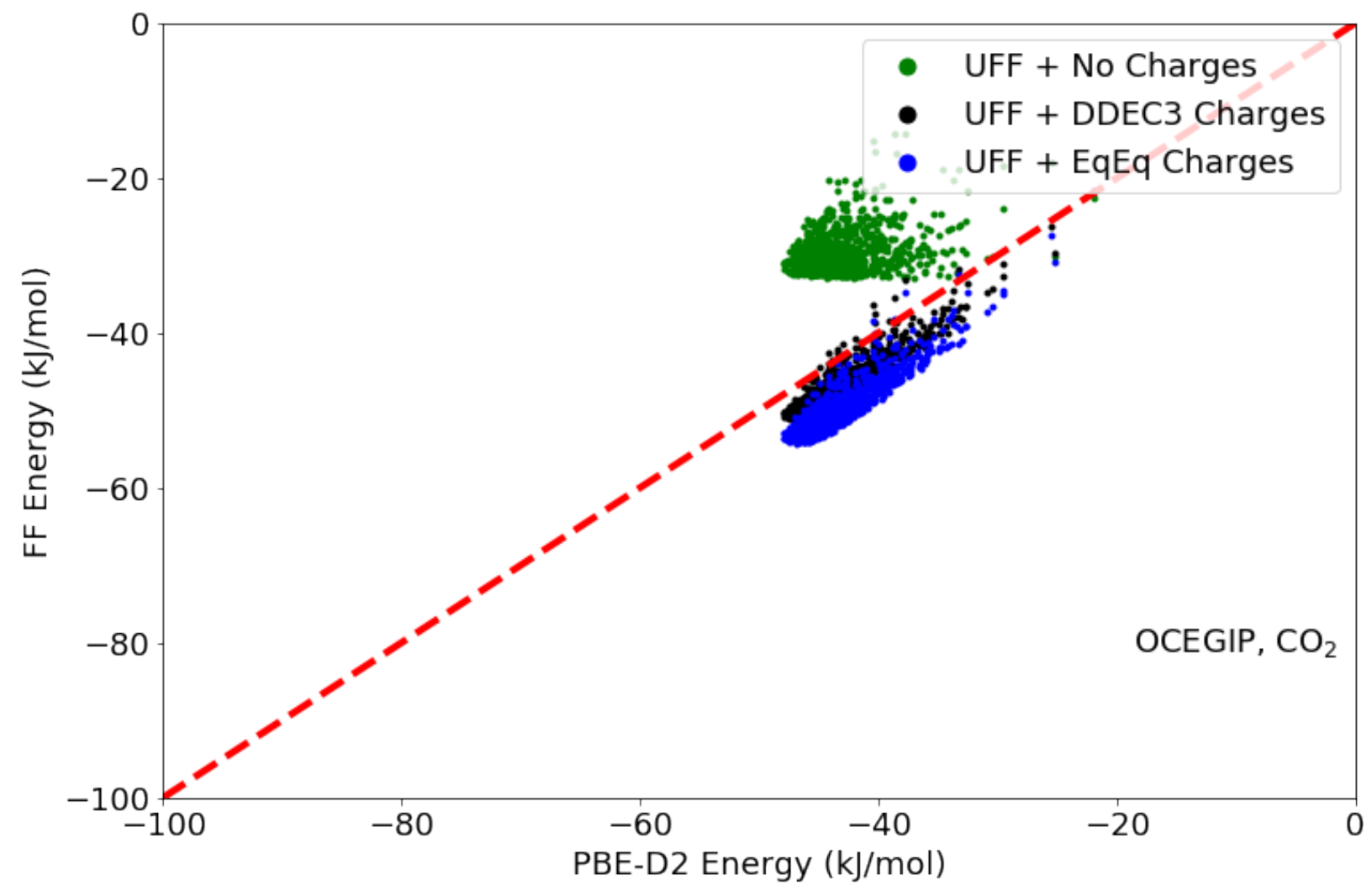

Figure S5 - A parity plot showing comparing interaction energies for PBE-D2 and UFF for $\mathrm{CO}_{2}$ in the MOF with structure code OCEGIP. Independent UFF calculations using no charges on MOF atoms (green), DDEC3 point charges on MOF atoms (black) and EqEq point charges on MOF atoms (blue) are shown. 


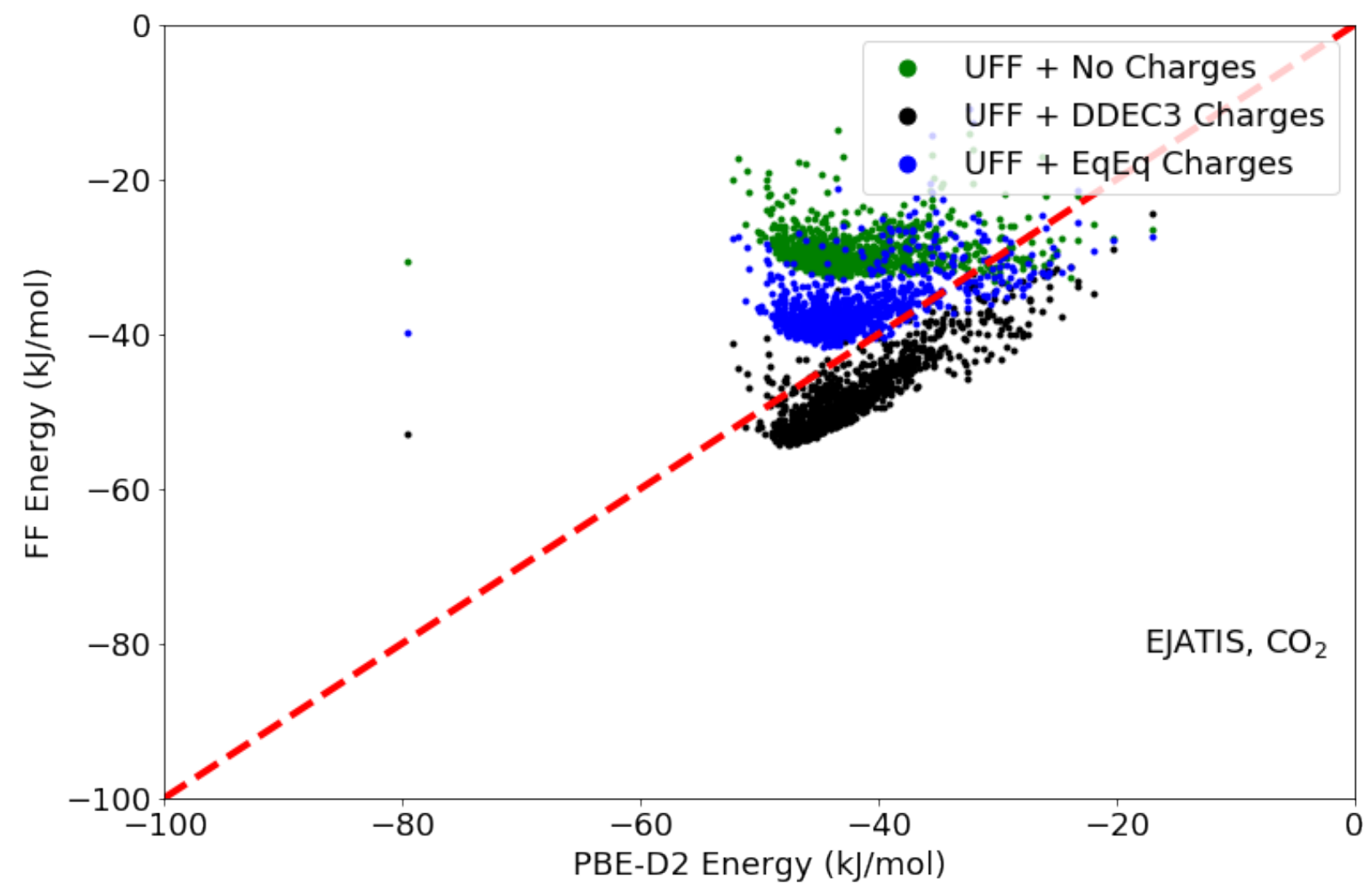

Figure S6 - A parity plot showing comparing interaction energies for PBE-D2 and UFF for $\mathrm{CO}_{2}$ in the MOF with structure code EJATIS. Independent UFF calculations using no charges on MOF atoms (green), DDEC3 point charges on MOF atoms (black) and EqEq point charges on MOF atoms (blue) are shown. 